\title{
hot off the press
}

\section{Endothelial laminins underlie the tip cell microenvironment}

\author{
Jan Kitajewski
}

$\mathrm{T}$ he concept of an endothelial 'tip cell', that leads the charge of a new vascular sprout, has captured the imagination of vascular biologists. The introduction of the tip cell concept allowed us to understand better the initial steps of sprouting angiogenesis (Gerhardt et al, 2003). This has spurred research at both academic and industry labs to harness knowledge of tip cell biology in order to manipulate tumour vasculature. Endothelial tip cells communicate with their neighbouring endothelial 'stalk' cells through a complex network of dominant and feedback signalling pathways that regulate endothelial growth and differentiation (Thurston \& Kitajewski, 2008). Tissue-derived VEGF promotes Notch ligand expression on endothelial tip cells, which then activate Notch and regulate VEGF receptors to define the endothelial stalk cell (Phng \& Gerhardt, 2009). This cellular communication mechanism does not occur in a vacuum. Instead, the differentiation of endothelial tip cells occurs in a matrix and multicellular milieu that I refer to as the 'tip cell microenvironment'. The Mettouchi group, earlier this year, and the Gerhardt lab - in this issue of EMBO reports-address how the basement membrane regulates tip cell behaviour (Estrach et al, 2011; Stenzel et al, 2011).

Approximately four decades ago, anatomists described the accumulation and arrangement of endothelial basement membrane material, and related the observed structural changes to distinct stages of sprouting angiogenesis (Bar \& Wolff, 1972). It was suggested that—during intensive capillary sprouting-"basement membrane plays a role in regulating differentiation and mitotic division of the adjacent cells" (Bar \& Wolff, 1972). Two fascinating new reports address this longheld speculation. The Mettouchi group describe the role of basement membrane components on cultured endothelial cell signalling (Estrach et al, 2011), whereas the Gerhardt lab pursued genetic analysis of mice deficient in laminin a4 to demonstrate a role for basement membrane in tip cell behaviour (Stenzel et al, 2011).

Biochemical and cellular analyses of integrins and their extracellular matrix (ECM) ligands has uncovered a myriad of cell-toECM communications with symphonic clarity (Avraamides et al, 2008). In complex mammalian tissues, analysing the function of integrins and their ligands in mutant mice is much less harmonic and more akin to orchestral tuning. In whole animals, looking in the right place at the right time might make all the difference. Gerhardt and colleagues focused on laminin $a 4$ and the heterotrimeric laminin 411 complex, consisting of laminins $\alpha 4, \beta 1$ and $\gamma 1$. Laminin $\alpha 4$ mutant mice are born anaemic due to embryonic bleeding and show aberrant responses during pathological angiogenesis (Thyboll et al, 2002). By focusing on retinal angiogenesis, they found that laminin a 4 is most strongly expressed in the leading tip cell. The phenotype of laminin a4 mutant mice suggested a link to Notch, as these mutant retinas have increased vascular density and excessive branching.

Exploring the link between Notch and laminin a4, Gerhardt and colleagues propose that laminin a 4 regulates DII4 in vivo, a regulation previously documented in cultured endothelial cells (Estrach et al, 2011). One subtle difference between the two studies addressing this hypothesis is that Stenzel and colleagues demonstrate regulation of Dll4 levels by laminin 411 both in vitro and in vivo, whereas Estrach and colleagues find that cultured human endothelial cells respond to laminin 111. In mice, laminin 411 is crucial, as shown from genetic analysis of laminin a4 mutant mice (Stenzel et al, 2011). Laminin 411 induced the expression of both D//4 and Vegfr-2 transcripts in mouse bEND5 endothelial cells, and blocking VEGFR-2 activity reduced laminin-411-mediated DII4 induction (Stenzel et al, 2011). Interestingly, in mosaic embryoid bodies containing wildtype and laminin a4-mutant embryonic stem cells, wild-type cells take over the role of basement membrane formation.

Using human umbilical venous endothelial cells (HUVEC) - commonly used for in vitro modelling of angiogenesis-Estrach and colleagues uncovered a lamininmediated, integrin signalling pathway that promotes DIl4 expression. Integrin $\alpha 2 \beta 1$ acts as a laminin receptor that suppresses endothelial cell proliferation, as does DII4/ Notch signalling. Overexpression of Dll4 in HUVEC reduced endothelial proliferation on fibronectin, an effect reminiscent of HUVEC grown on laminin. Conversely, DII4 knockdown partly rescued the minimal cell proliferation seen when HUVEC are grown on laminin. This implies that laminin affects DII4, which activates Notch signalling leading to suppression of endothelial proliferation. In HUVEC, integrin $\alpha 2$ is a key adhesion receptor for laminin and was also found to be required for DII4 induction by laminin. Thus, one can propose that laminin induces DIl4 using integrin $\alpha 2 \beta 1$-mediated signalling. Which transcriptional pathway mediates this DII4 induction? Although the upstream sequence of DII4 is full of potential transcription regulatory elements, previous studies highlighted FoxC2 as an important regulator of Dll4 in arterial endothelium. This also seems to be the case in cultured HUVEC, where FoxC2 is transiently and specifically induced by laminin, and its knockdown results in impaired induction of DII4 by laminin. Further strengthening the argument for 


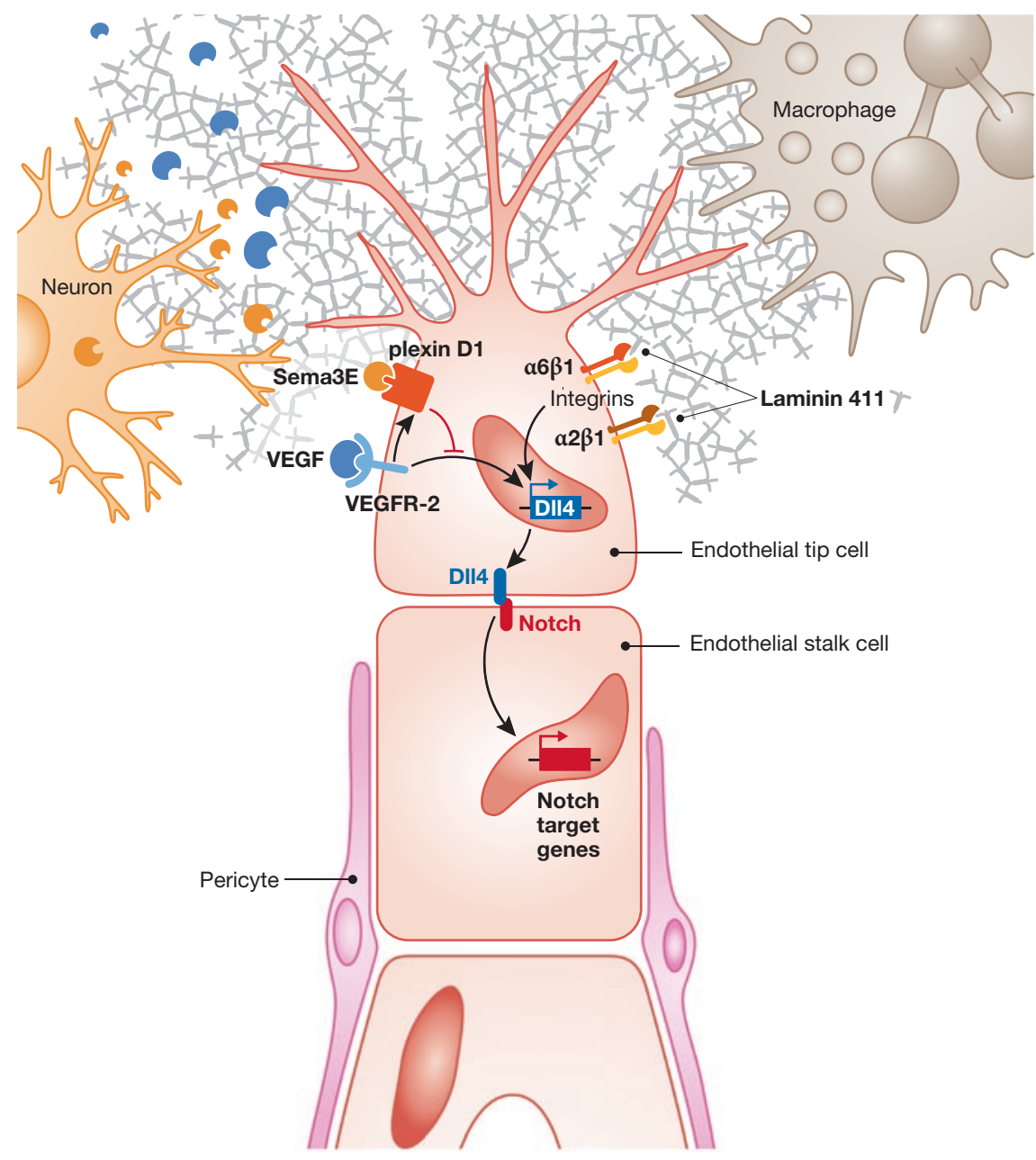

Fig 1 | The tip cell microenvironment. Endothelial tip cells respond to both VEGF, through VEGFR-2, and laminin, through integrin $\alpha 2 \beta 1$ and $\alpha 6 \beta 1$. VEGF and laminin-mediated signals induce Dll4, which activates Notch proteins in neighbouring stalk cells. Other regulators of basement membrane components that act in the tip cell microenvironment include macrophages, neurons and pericytes.

this circuitry, the Mattouchi lab show that knockdown of integrin a2 impairs the induction of FoxC2 by laminin. This model posits that laminin promotes integrin a $2 \beta 1$ signalling, thereby inducing FoxC2, a transcription factor that induces DII4, thus activating Notch signalling. Whether FoxC2 acts in parallel with the VEGFR-2 pathway to induce DIl4 is not clear.

How does the laminin-integrin-DII4/ Notch axis work during retinal angiogenesis? The Gerhardt group studied this comprehensively by using mouse mutant analysis. Endothelial cells need $\alpha 2, \alpha 3$ and $\alpha 6$ integrins to interact and adhere to laminins and $\alpha 2$ or a6 to allow for laminin-mediated induction of Dll4. Turning to the retina, they did not see a hyper-sprouting (aka Notch-related) phenotype in mice with global gene deficiencies for integrins $\alpha 2, \alpha 3$ or $\beta 3$. So where does one go from there? Endothelial-specific loss of integrin $\beta 1$, a partner for $\alpha 2$ or $a 6$ subunits, resulted in reduced retinal DII4 expression and hypersprouting of mutant retinas. Considering their previous results implicating laminins in tip cell phenotypes, this highly informative outcome brings the model full circle.

Therefore, during sprouting angiogenesis, tip cells can produce laminins, which in turn act through integrins to regulate the Notch signalling pathway. Specifically, murine laminin 411 induces Vegfr-2 and D//4, which activates Notch signalling in endothelial cells (Fig 1). The induction of D/l4 in endothelial cells requires integrin $\beta 1$ in vivo, and depends on integrins $\alpha 2$ or $\alpha 6$ in vitro. Taken together, Dll4 regulation by laminin 411 probably involves both $\alpha 2 \beta 1$ and $\alpha 6 \beta 1$. Thus, tip cells influence and respond to a dynamic microenvironment that determines whether they will maintain their leading characteristics or differentiate into their altered state, that of an endothelial stalk cell. The instruments that perform in this orchestra are specific basement membrane components, integrins, VEGF receptors, Notch ligands, Notch proteins and Notch target genes.

What have we learned about the tip cell microenvironment? Macrophages_long known to influence basement membrane composition — can respond to tip cell signals designed to activate macrophage Notch proteins (Outtz et al, 2011) and might influence tip cell behaviour. Furthermore, neurons can influence the DIl4/Notch pathway. Gu and colleagues have recently reported that VEGF controls the expression of plexin D1, the receptor for semaphorin 3E (Sema3E; Kim et al, 2011). In the murine retina, plexin D1 is primarily expressed in endothelial cells at the front of sprouting blood vessels. Sema3E is secreted by retinal neurons allowing for Sema3E-plexin D1 signalling in endothelial cells. This is yet another regulatory pathway affecting Dll4 expression, as "gain and loss of function of Sema3E and Plexin-D1 disrupts normal Dll4 expression, Notch activity, and tip/stalk cell distribution in the retinal vasculature" (Fig 1; Kim et al, 2011).

With so many conductors directing the nascent sprout, including basement membrane, macrophages and neurons, in addition to other endothelial cells, how does the tip cell keep things straight? That is one of the marvels of nature.

\section{REFERENCES}

Avraamides CJ, Garmy-Susini B, Varner JA (2008) Nat Rev Cancer 8: 604-617

BarT, Wolff JR (1972) Z Zellforsch Mikrosk Anat 133: 231-248

Estrach S et al (2011) Circ Res 109: 172-182 Gerhardt H et al (2003) / Cell Biol 161: 1163-1177 Kim J et al (2011) Genes Dev 25: 1399-1411 Outtz HH et al (2011) Blood 118: 3436-3439 Phng LK, Gerhardt H (2009) Dev Cell 16: 196-208 Stenzel D et al (2011) EMBO Rep [Epub ahead of print] doi:10.1038/embor.2011.194

Thurston G, Kitajewski J (2008) Br J Cancer 99: 1204-1209

Thyboll J et al (2002) Mol Cell Biol 22: 1194-1202

Jan Kitajewski is at the Departments of Obstetrics/Gynecology and Pathology, Herbert Irving Comprehensive Cancer Center, Columbia University, New York, USA. E-mail:jkk9@columbia.edu

Published online 21 October 2011

EMBO reports (2011) 12, 1087-1088.

doi:10.1038/embor.2011.202 